\title{
Enfoque de capacidades y ética profesional en la formación universitaria para la democracia: el caso de las carreras de negocios y humanidades de una universidad privada en México
}

\author{
Capabilities approach and professional ethics in university education for democracy: \\ the case of business and humanities careers at a private university in Mexico \\ Juan Martín López-Calva \\ Claudia Rebeca Méndez Escarza
}

\begin{abstract}
RESUMEN
Esta investigación se sustenta en la aportación teórica del enfoque de capacidades de la filósofa estadounidense Martha Nussbaum como fuente de criterios éticos de la formación universitaria que orienten la formación profesional hacia la construcción de sociedades auténticamente humanas y con su conceptualización de distinción entre lo que llama educación para la renta y educación para la democracia. A partir de estos rasgos se construyó una guía de entrevista semiestructurada que fue aplicada a una muestra de ocho académicos de una universidad privada, quienes tienen a su cargo la dirección de programas de licenciatura en las áreas de Negocios y de Humanidades, para conocer la manera en que conciben y están llevando a la práctica la formación de futuros profesionistas en sus respectivas disciplinas. Se encontraron algunas diferencias en la comparación entre ambas áreas, pero los resultados muestran en general una orientación hacia la educación para la democracia en las carreras de ambos campos disciplinares, con un mayor énfasis y claridad en las de Humanidades, lo cual puede deberse a la comprensión y adhesión al ideario y la misión de la universidad en la que se realizó el estudio o al uso de un discurso institucional aprendido.
\end{abstract}

Palabras clave: bien común, educación superior, enseñanza técnica, formación de profesionales, humanismo.

\begin{abstract}
This research is based on the theoretical contribution of the capabilities approach of American philosopher Martha Nussbaum as a source of ethical criteria of university education that guide professional training towards the construction of authentically human societies and her distinction between what she calls education for profit and education for democracy. Based on these features, a semi-structured interview guide was constructed and later applied to a sample of eight academics from a private university, who are in charge of directing undergraduate programs in the areas of Business and Humanities, in order to learn how do they conceive and are putting into practice the training of future professionals in their respective disciplines. Some differences were found in the comparison between both areas, but the results generally show an orientation towards education for democracy in the careers of both disciplinary fields with a greater emphasis and clarity in those of Humanities, which may be due either to the understanding and adherence to the ideology and mission of the university where the study was carried out or the use of a learned institutional discourse. Keywords: common good, higher education, technical education, professional training, humanism.
\end{abstract}




\section{INTRODUCCIÓN}

La hegemonía del sistema económico basado en la producción y el consumo ilimitado de bienes materiales definido con el término "neoliberalismo", que se profundizó y extendió por todo el planeta con el fenómeno de la globalización que paradójicamente permite el libre flujo de mercancías derribando las fronteras nacionales pero bloquea el tránsito libre de personas en la búsqueda de mejores condiciones de vida, ha tenido consecuencias en todos los campos de la vida humana en las décadas recientes.

La educación universitaria no ha estado ajena a este fenómeno y ha sido cada vez más exigida por el mercado para formar profesionistas competitivos y eficientes que se conviertan en meros repuestos de la fuerza de trabajo para el mercado laboral, convirtiendo la educación universitaria en un proceso de capacitación técnica de alto nivel que excluye elementos que son esenciales para que una educación pueda llamarse "universitaria".

La educación enfocada desde una visión práctica y de aplicación inmediata del conocimiento se ha vuelto una mercancía más en el mercado de bienes y servicios del sistema capitalista neoliberal global, en un proceso que investigadores como Brunner (2006) han llamado "mercadización de la educación superior".

En la sociedad del conocimiento, las instituciones generadoras, transmisoras e innovadoras del conocimiento están viviendo una tensión permanente entre la conservación de la esencia que constituye la vida universitaria desde su fundación y desarrollo histórico (Borrero, 2008) y la adaptación responsiva a un mercado que exige, cada vez más, egresados capacitados para la competencia descarnada y la autoexplotación (Han, 2017), que implica obediencia, ausencia de crítica al sistema y exclusión de la formación ética profesional que todo universitario debería adquirir en su proceso formativo (Cortina, 2000).

En su libro Sin fines de lucro, la filósofa estadounidense Martha Nussbaum (2010) llama a este tipo de formación sustentada en la meta única del incremento del producto

Juan Martín López-Calva. Decano de Artes y Humanidades de la Universidad Popular Autónoma del Estado de Puebla, México. Es doctor en Educación por la Universidad Autónoma de Tlaxcala. Ha realizado dos estancias postdoctorales en el Lonergan Institute de Boston College y publicado treinta libros, cuarenta y cinco artículos y más de treinta capítulos de libros. Es miembro del Sistema Nacional de Investigadores, del Consejo Mexicano de Investigación Educativa, de la Red Nacional de Investigadores en Educación y Valores, y de la Asociación Latinoamericana de Filosofía de la Educación. Trabaja en las líneas de educación humanista, pensamiento complejo, ética profesional y educación en valores. Correo electrónico: juanmartin.lopez@ upaep.mx. ID: https://orcid.org/0000-0002-6948-8556.

Claudia Rebeca Méndez Escarza. Universidad Popular Autónoma del Estado de Puebla, México. Es diseñadora de la Comunicación Gráfica por la UAM-Xochimilco y maestra en Teoría del Diseño por la UDLAP. Certificada como profesora de inglés. Actualmente estudia el doctorado en Diseño y Visualización de la Información en la UAM-Azcapotzalco. Se desempeña como directora académica de la Facultad de Diseño en UPAEP. Imparte clases en licenciatura y posgrados en el área del diseño de información, el diseño estratégico y el diseño centrado en la persona. Correo electrónico: claudiarebeca.mendez@upaep.mx. ID: https://orcid.org/0000-0002-8887-3257. 
interno bruto (PIB) de los países "Educación para la renta", y plantea la disyuntiva entre este tipo de formación técnica y una formación integral que incluya las artes, las humanidades y por supuesto la ética para desarrollar no solamente empleados sino ciudadanos capaces de construir y mantener sociedades democráticas.

El exrector de la Universidad Centroamericana (UCA) de Nicaragua, Xabier Gorostiaga S. J., habló en un muy duro pero realista discurso de graduación de su institución de que las universidades en el contexto del mundo injusto y excluyente del capitalismo salvaje están formando "profesionales exitosos para sociedades fracasadas” (José María Tojeira, citado en ETEA, s.f.).

¿Cómo viven los directores de licenciaturas de las áreas de Negocios y de Humanidades esta tensión entre una educación para la renta que exige el mercado laboral y la educación para la democracia con sentido ético profesional que se requiere para construir otro mundo posible en este cambio de época?

El presente trabajo de investigación reporta los resultados de entrevistas semiestructuradas aplicadas a ocho directores de programas académicos de ambas áreas disciplinares en una universidad privada del centro de México que se define en su ideario como humanista y de identidad católica.

El proyecto de investigación partió de dos supuestos centrales:

1. La tensión entre educación para la renta y educación para la democracia está presente en la gestión de la formación universitaria, sobre todo por tratarse de una universidad particular.

2. Por la filosofía de la institución y el discurso que se enfatiza sistemáticamente entre los colaboradores y hacia la sociedad, es probable que haya una mayor inclinación hacia la educación para la democracia.

\section{El enfoque de capacidades como visión ética de las profesiones}

...el gran reto del tercer Milenio consistirá, a mi juicio, en diseñar una idea de felicidad que incluya, como un componente suyo ineludible, el afán de justicia. Hemos depauperado excesivamente la felicidad, la hemos dejado en elemental bienestar, en estar bien, en tener lo suficiente. Somos muy modestos y no nos atrevemos a hablar de felicidad, sino, a lo sumo, de calidad de vida: llevar una vida de calidad, todo pequeñito, modesto, poco ambicioso. Y, sin embargo, es preciso recuperar la aspiración a la felicidad. Decía Aristóteles, hace ya veinticuatro siglos, que todos los seres humanos tienden a la felicidad, y hubiera sido igualmente verdad aunque no lo hubiera dicho: todos los seres humanos tienden a la felicidad, y no podemos arrojar la toalla en esto, tenemos que diseñar una idea de felicidad, que tenga como componente ineludible la justicia [Cortina, 2000-2001, p. 1].

El gran desafío de la ética aplicada a la vida humana y social pero también en el campo concreto de las profesiones es el de la conjunción de las condiciones para que cada uno pueda lograr la felicidad que anhela y al mismo tiempo existan las condiciones estructurales de justicia sin las cuales es imposible alcanzar esta idea de felicidad. 
Todo el mundo quiere ser feliz, pero todo el mundo debe tratar de ser justo, dice la misma Cortina (2000-2001) acerca de estos dos pilares sobre los cuales se propone construir la ética que esté a la altura de los tiempos que vivimos y responda a los desafíos del mundo contemporáneo.

Porque en este mundo del mercado global se ha ido imponiendo la idea de que la felicidad puede prescindir de la justicia, porque se ha construido una idea de felicidad que por una parte es individualista y por otra se equipara al concepto de nivel de vida. Entre más alto nivel de vida se tenga más feliz se va a ser en la vida, sin importar si existen millones de seres humanos que no tienen lo indispensable para sobrevivir y menos pueden aspirar a vivir y a construir su proyecto de felicidad.

Se trata de una ética del bienestar individual que por más que se haya inoculado en el ADN del ser humano contemporáneo no alcanza a satisfacer su búsqueda auténtica de humanización (Morin, 2003). Esta perspectiva de la felicidad individual entendida como nivel de vida se ha venido introyectando en las instituciones de educación superior que hoy compiten por formar a los "mejores profesionales del mundo", sin importar, como afirmaba Gorostiaga en la cita ya planteada, el fracaso social y la ruptura del tejido de convivencia en el que tengan que vivir.

Esta visión hace necesaria la búsqueda de propuestas teóricas que traten de aportar elementos para construir una visión ética aplicada a la búsqueda de formas de vida y estructuras que concilien la visión y aspiración de felicidad integral y profunda connatural a todo ser humano con la construcción de estructuras sociales justas que hagan posible esta felicidad.

Una respuesta muy pertinente y sólidamente fundada para la adopción de esta visión ética que concilie felicidad con justicia es el llamado enfoque de capacidades, desarrollado en su dimensión filosófica social por Martha Nussbaum (1997, 2010, 2012) y en sus aplicaciones a la economía en el combate a la pobreza en el mundo por Amartya Sen (1999, 2009).

Desde nuestro punto de vista, el enfoque de capacidades es compatible con la ética profesional de principios (Beauchamp y Childress, 2001; Hortal, 2002) puesto que, en primer lugar se concibe como una teoría de la justicia -principio de justiciaque busca promover el bienestar y el bien vivir de las sociedades democráticas enfatizando la necesidad de dotar de capacidades a los más pobres para que mejoren sus condiciones de vida -principio de beneficencia-, considerándolos como sujetos con dignidad propia y derechos a respetar y no como simples receptores de programas de compensación o subsidio social -principio de autonomía-.

Según Nussbaum (2012), “una capacidad es aquello que responde a la pregunta básica por ¿Qué es lo que puede una persona o grupo social hacer o ser? y ¿Qué oportunidades reales tiene para lograrlo?” (p. 18). Para la filósofa estadounidense existen capacidades internas que son propias de la persona y capacidades combinadas que tienen que ver con el entorno y sus condiciones estructurales y culturales. 
Por ejemplo, para poder desarrollarse humanamente una persona tiene que desarrollar su capacidad interna de pensamiento y expresión, pero de nada sirve desarrollarla si en el entorno sociopolítico en el que vive no hay condiciones ni libertad para pensar por uno mismo y expresar lo que se piensa.

Tanto Nussbaum (2012) como Sen (1999) coinciden en que la capacidad equivale a libertad para hacer realidad ciertos funcionamientos de las personas y los grupos en su contexto social, ya que un individuo es más libre si tiene mayores oportunidades de elegir entre varias opciones de vida.

"Las personas deben ser vistas [...] como agentes activamente involucrados [...] en la construcción de su propio destino y no solamente como receptores pasivos del fruto de ingeniosos programas de desarrollo" (Sen, 1999, p. 53). Esta afirmación incluye a los futuros profesionistas que requieren ser formados en el desarrollo continuo de sus capacidades de elección entre opciones de ejercicio profesional en la sociedad y no como futuros repositorios de cuadros obedientes al sistema que les tiene ya diseñada una sola opción para vivir.

Nussbaum (2012, pp. 33-34) además menciona -cosa en la que Sen (1999) no coincide- un listado de capacidades fundamentales que toda sociedad democrática debería garantizar para todas las personas en su desarrollo. Estas capacidades son:

- Vida: poder vivir hasta el final una vida normal, no morir prematuramente por enfermedades curables o violencia.

- Salud física: tener una nutrición y acceso a un sistema de salud de calidad.

- Integridad corporal: contar con un gobierno que garantice la seguridad y la integridad sin sufrir agresiones.

- Sentidos, imaginación y pensamiento: desarrollar la capacidad sensorial, la imaginación creativa y el pensamiento propio a partir de una buena educación.

- Emociones: desarrollar las propias emociones de manera sana e integrada.

- Razón práctica: ser capaz de desarrollar y vivir una concepción del bien. Esta capacidad es la capacidad ética por excelencia.

- Afiliación: ser capaz de una sana forma de vivir con otros, de establecer relaciones humanas constructivas e incluyentes.

- Relación con otras especies: tener una relación armónica con la naturaleza y las otras especies animales.

- Juego: tener la oportunidad y los espacios para desarrollar la dimensión lúdica y el tiempo adecuado para la diversión como parte del desarrollo humano.

- Control sobre el entorno político y material: poder participar mediante el voto y la organización y expresión libre en una sociedad democrática y tener garantizado el derecho de propiedad de bienes materiales.

Como puede apreciarse a través de esta síntesis breve que no se desarrolla más por razones de espacio, el enfoque de capacidades no solamente es compatible con la 
ética de principios, sino que plantea una concepción del ser humano y su desarrollo en libertad efectiva (Lonergan, 1988) para poder elegir su proyecto de felicidad en una sociedad que tenga las condiciones de justicia -capacidades básicas- para lograrlo.

Este desarrollo de capacidades se puede realizar en un entorno de educación superior que se sustente en visiones humanistas y democráticas que trasciendan la perspectiva hegemónica de la mercadización de la educación superior que hoy se ha venido imponiendo en el mundo. A esto responde la concepción de dos visiones de educación que plantea Nussbaum (2010), que son las de la educación para la renta y de la educación para la democracia, que desarrollaremos brevemente en el siguiente apartado.

\section{Educación para la renta y educación para la democracia: rasgos centrales}

En su célebre discurso al recibir el doctorado honoris causa por la Universidad de Antioquia en Colombia, la filósofa Martha Nussbaum (2015) afirma que el mundo está en una crisis de grandes proporciones y que no se refiere a la crisis económica que estalló en el 2018, ni a la crisis provocada por el terrorismo internacional en expansión, sino a una crisis que parece pasar desapercibida para la mayoría de la población y que puede causar mayores daños en el largo plazo para el mundo: se trata de una "crisis mundial en la educación" (p. 1).

"Ansiosas de lucro nacional, las naciones y sus sistemas de educación están descartando descuidadamente habilidades que son necesarias para mantener vivas las democracias", afirma en el mismo texto, y plantea que de continuar esta tendencia el mundo pronto tendrá generaciones de máquinas útiles, "en lugar de ciudadanos completos que puedan pensar por sí mismos, criticar la tradición y entender el significado de los sufrimientos y logros de otra persona”, es decir, profesionales con una ética aplicada a su ejercicio que tomen decisiones responsables a partir del pensamiento crítico y de la búsqueda del bienestar propio y sobre todo del bien de la sociedad.

Es por ello que planteamos en el apartado anterior la visión del enfoque de capacidades como una perspectiva teórica que tiene como eje la ética profesional, totalmente convergente con los principios de beneficencia, autonomía y justicia (Hortal, 1996).

Nussbaum (2010) llama a esta tendencia errónea que está produciendo la crisis mundial "Educación para la renta", puesto que su objetivo es capacitar profesionales empleables y eficientes en el mercado laboral pero no ciudadanos libres, críticos y responsables.

A esta tendencia opone lo que llama "Educación para la democracia", que es una formación completa que tiene un sustento ético profesional y una visión social orientada a la construcción del bien común.

En la tabla 1 enlistamos los principales rasgos que la filósofa estadounidense considera distintivos de estos dos enfoques. 
Tabla 1. Características de la educación para la renta y para la democracia.

Educación para la renta

Propósito de la educación con fines de crecimiento económico

Educación puramente científica o técnica, excluyendo a las humanidades

Aptitudes necesarias: alfabetización y competencias matemáticas

Conocimientos avanzados en informática

La igualdad de acceso a la educación no es tan importante

Exclusión del pensamiento crítico

No hay libertad de pensamiento en el estudiante sino obediencia, capacitación y operación

Preponderancia del nacionalismo sobre la justicia social

Desprecio (y miedo) por el arte y la literatura
Educación para la democracia

Aptitud para reflexionar sobre las cuestiones políticas Aptitud para reconocer al otro como persona con los mismos derechos

Interesarse por la vida del otro (comprensión) Asumir la complejidad de la existencia humana Aptitud de juicio crítico sobre los dirigentes políticos Pensar en el bien común de la nación como un todo Aptitud para concebir a la propia nación como parte de un orden mundial complejo

Fuente: Construcción personal basada en Nussbaum (2010).

Como se puede ver en la tabla 1, la educación para la renta excluye -por considerarlas inútiles, pero también porque les teme- a las artes, las humanidades, el desarrollo del pensamiento crítico, de la conciencia histórica y de la empatía hacia los demás a partir del reconocimiento y respeto de su vida, elementos todos que son parte de toda ética y, como dijimos líneas atrás, se corresponden con los principios de la ética profesional.

Se trata de una educación meramente técnica que habilita en matemáticas, tecnologías de información y comunicación (TIC), finanzas y disciplinas prácticas y consideradas útiles para la contribución del profesionista al crecimiento económico del país.

Por el contrario, la educación para la democracia, en lugar de excluir la formación en artes y humanidades, la incluye como parte importante de la preparación profesional y ciudadana. Como diría Edgar Morin (2000), en vez de sacrificar estas disciplinas, las magnifica y las mantiene vivas, no como un cofre de cenizas al que hay que rendir culto sino como un fuego que hay que mantener encendido, según la famosa frase que algunos atribuyen a Gustav Mahler: "La tradición es la transmisión del fuego y no la adoración de las cenizas” (El País, 2010, párr. 2).

Es lógico suponer, desde una mirada no simplificadora del fenómeno de la educación superior, que no hay instituciones o países que tengan una educación para la renta exclusivamente y otros que desarrollen en estado puro la educación para la democracia, sino que hay una tensión entre el mundo contemporáneo dominado por la visión economicista que empuja hacia esta crisis educativa para la renta y el mundo universitario con una larga tradición humanista reforzada por las grandes declaraciones internacionales sobre derechos humanos y democracia, que busca mantener encendido el fuego de la educación para la democracia. 
Esta tensión es la que se buscó indagar en el proyecto de investigación que ahora reportamos.

\section{Notas METOdológicas}

La investigación aquí reportada se realizó desde una visión cualitativa de corte hermenéutico (Cárcamo, 2005) que buscó indagar y caracterizar las experiencias y significados que los directores académicos de las áreas de Negocios y de Humanidades de una institución de educación superior de financiamiento privado tienen acerca de la tensión actual entre la educación para la renta y la educación para la democracia, tanto en la forma en que perciben esta tensión en el estado actual de sus disciplinas como en la manera en que esta tensión se refleja en la formación de los futuros profesionistas y cómo enfrentan esta tensión si es que la viven.

Como afirma Cárcamo (2005):

Puntualmente el análisis hermenéutico se enmarca en el paradigma interpretativo comprensivo; lo que supone un rescate de los elementos del sujeto por sobre aquellos hechos externos a él. En este sentido, debe destacarse que dicho análisis toma como eje fundamental el proceso de interpretación [p. 211].

La unidad de análisis, por tanto, fueron los directores académicos de programas de licenciatura de estas dos áreas disciplinares, ocho de un total de quince en ambas decanaturas, cuatro del área de Artes y Humanidades y cuatro de la de Negocios, que constituyeron una muestra de casos-tipo que se utilizan cuando el "objetivo es la riqueza, profundidad y calidad de la información, no la cantidad ni la estandarización. En estudios con perspectiva fenomenológica, donde el objetivo es analizar los valores, ritos y significados de un determinado grupo social, el uso de muestras tanto de expertos como de casos-tipo es frecuente" (Hernández, Fernández y Baptista, 2010, p. 397).

Siete de los ocho sujetos entrevistados tienen grado de doctorado concluido y uno de ellos está actualmente cursando sus estudios doctorales. Todos tienen más de cinco años de trabajo en la universidad, la mitad de ellos tienen más de diez. En cuanto a la antigüedad en el puesto, los directores del área de Humanidades tenían en el momento de la entrevista un año en el cargo y los del área de Negocios cinco años en promedio al frente de sus programas académicos. Todos los directores del área de Negocios que fueron entrevistados han tenido experiencia de entre cinco y diez años en empresas antes de trabajar como académicos, y solamente una directora del área de Artes y Humanidades reportó tener experiencia de trabajo en empresa, previa a su ingreso a la universidad.

El instrumento fue una guía de entrevista semiestructurada compuesta por una primera sección de datos censales (grado académico, lugar donde realizó sus estudios, antigüedad en la universidad, antigüedad como director de programa académico, 
años como académico en total y, si la tuvieran, años de experiencia profesional en empresas, gobierno u otras organizaciones distintas a la universidad) y un conjunto de siete preguntas abiertas.

Se siguió un protocolo ético y se pidió a todos los entrevistados firmar una carta de consentimiento informado y, por otra parte, autorizar la grabación en audio de la entrevista. La duración de la entrevista osciló entre los 30 y los 40 minutos.

Se procedió primero explicando a través de un correo electrónico personalizado el objetivo y las características de la investigación y solicitando la anuencia para participar en la entrevista. Una vez que los sujetos aceptaron ser entrevistados, se concertó una cita a través del mismo correo electrónico. Todas las entrevistas se realizaron en el cubículo de cada director académico directamente por los dos coautores de este trabajo.

Como ya se dijo, el contexto de la investigación fue una universidad privada que se define como humanista y de identidad católica. Está ubicada en una ciudad capital importante en el centro de la República mexicana y tiene alrededor de diez mil estudiantes de licenciatura y aproximadamente siete mil más entre sus bachilleratos y sus distintos programas de posgrado.

Por su propia definición filosófica, la universidad incluye en sus planes de estudio un área de asignaturas de formación humanista y diversas experiencias en un amplio espectro en espacios cocurriculares y extracurriculares de carácter social, espiritual, artístico, filosófico y cultural.

Su estructura organizacional está basada en decanaturas que agrupan a distintas escuelas y facultades desde las que se ofrecen los programas de licenciatura, posgrado y educación continua y se realizan proyectos de investigación y actividades de difusión del conocimiento.

El marco de interpretación de los datos fue de corte fenomenológico, puesto que se buscó entender los significados de la experiencia de cada director académico de licenciatura en el tema de estudio a través del análisis del discurso y la búsqueda de convergencias y divergencias en las perspectivas de cada uno de ellos.

La idea fue explorar en:

1) Las percepciones, desde la experiencia personal, de la existencia de la tensión entre educación para la renta y educación para la democracia en la formación de sus estudiantes.

2) Los elementos centrales de la vida cotidiana en la formación de los profesionistas de sus disciplinas, buscando aquello que consideran más significativo e interesante.

El proceso para procesar los datos se hizo de forma manual mediante una tabla de Excel en la que se construyó una matriz que tenía en el eje vertical los principales rasgos de la tabla 1 que hemos presentado sobre educación para la renta y educación 
para la democracia y en el eje horizontal las respuestas de los ocho sujetos. Se hizo una matriz por cada una de las preguntas.

\section{RESUltados DE LAS ENTREVISTAS}

Presentamos a continuación una síntesis de los resultados de las entrevistas realizadas. Hacemos un ejercicio de análisis sintético de las primeras cuatro preguntas y, por cuestiones de espacio y de convergencia, de las últimas tres preguntas a la institución donde se realizó la investigación; planteamos un análisis integrado de estas preguntas finales. Es conveniente iniciar clarificando que los sujetos del 1 al 4 corresponden a directores del área de Negocios y los sujetos del 5 al 8 corresponden a la de Humanidades.

\section{Pregunta 1: rasgos que debe tener la educación profesional actual}

La mayoría de los directores entrevistados mencionó como características fundamentales en la educación universitaria actual elementos relacionados con contenidos de los que Nussbaum relaciona con la educación para la renta, como conocimiento del idioma inglés, de computación y de las bases técnicas y científicas de su profesión.

Sin embargo, esta pregunta -que se realizó previamente a darles a conocer la tipología de Nussbaum con sus respectivos rasgos- fue la que mostró mayor diferencia en las respuestas de los directores, ya que los directores del área de Negocios enfatizaron mucho más claramente elementos de educación para la renta y los de Humanidades se inclinaron más hacia la educación para la democracia.

Todos describieron rasgos de educación para la renta, pero los de Humanidades mencionaron además rasgos relacionados con una formación ética profesional entendida desde esta visión de educación para la democracia, por ejemplo: saber los conocimientos pero tener la capacidad de cuestionarlos críticamente; poder conocer no solamente el qué de la profesión sino las razones y las finalidades de cada elemento que se aprende; aplicar socialmente en beneficio del bien común lo aprendido, etc.

Una breve muestra de esta diferencia en las respuestas está en que, por ejemplo, el sujeto 1 enfatizó el conocimiento técnico de software, el trabajo en equipo, la estadística y el dominio del inglés como fundamentales, y no hizo ninguna referencia a rasgos relacionados con educación para la democracia, mientras que el sujeto 5 mencionó el dominio de las bases disciplinares de carácter antropológico y psicológico necesarias para la profesión, pero declaró también:

La integralidad de la persona es un paso sumamente importante. Cuáles son esas necesidades, cuál es ese entorno en el que vive, ese medio social también que es necesario ver por qué podemos, poder entender a la persona y estar en posibilidad de apoyarlo, ¿no?, de acompañarla en sus procesos. Por lo tanto, el conocimiento de la persona humana, el conocimiento y sobre todo las... pues la postura desde una mirada humanista [S5, P1]. 


\section{Pregunta 2: rasgos de la educación para la democracia que incluyen en sus programas y forma de trabajarlos}

En esta pregunta se manifiesta más homogeneidad porque se pregunta por rasgos de la educación para la democracia específicamente y se pide que se plantee la forma en que se trabajan en sus disciplinas.

En el área de Negocios destacan respuestas como:

Tenemos nosotros una asignatura que se llama Dirección de Organizaciones Civiles. En esa asignatura se trabaja mucho a los grupos vulnerables y sobre todo su inclusión. Muchos de los proyectos que se desarrollan ahí se trabajan a través de las políticas públicas [S1, P2].

Aquí se muestra que al tratarse de una universidad que se define como humanista y orientada al bien común de la sociedad, el diseño de planes de estudio incluye asignaturas de la profesión que se orientan hacia el vínculo o el aprendizaje-servicio con poblaciones vulnerables.

El sujeto 2, también del área de Negocios, habla de algo más transversal, de intenciones que se tratan de suscitar en todos los profesores, como:

...tratar de estar más pendiente de los demás y tener aptitud de juicio crítico [S2, P2].

En el área de Humanidades por supuesto hubo respuestas abundantes en este rubro de la educación para la democracia. Ponemos aquí un par de ejemplos de lo más significativo que se obtuvo en las respuestas, aunque no reflejan toda la riqueza de estas.

El sujeto 6, por ejemplo, habla más en términos de la universidad en general, aunque también respondió rasgos que tienen que ver con el currículo de la licenciatura que dirige. Su respuesta dice:

Creo que incluso no solo empatan las Humanidades, sino el ámbito universitario, la universitas, es un libre pensamiento donde se respetan todo tipo de pensamientos a nivel de cosmovisiones, dimensiones espirituales, apetitos, deseos; perfecto, pero con base científica, donde no hay que ofenderse [S6, P2].

Los sujetos 7 y 8 coinciden en la relevancia del rasgo de interesarse en la vida del otro como algo fundamental en la formación de sus estudiantes universitarios. El sujeto 8 lo declara de esta forma:

El tema de interesarse por la vida del otro yo creo que también, eh, uno de los temas que tenemos de investigación muy fuerte aquí en la universidad es el tema de la alteridad y el reconocimiento $[\mathrm{S} 7, \mathrm{P} 2]$.

\section{Pregunta 3: rasgos de educación para la renta y forma de trabajarlos}

En estos rasgos, los sujetos 1 y 2 del área de Negocios coinciden en la importancia del razonamiento matemático como algo central para la formación de los profesionales de la actualidad: 
Nosotros sí damos mucha importancia al tema matemático porque creemos que ese razonamiento ágil, matemático, puede ayudar a la solución de problemas, ¿no? [S2, P3].

En el área de Humanidades hay menos amplitud de respuestas en esta pregunta concreta. El sujeto 7 responde más bien hablando de la importancia de formar a sus estudiantes para no pensar en una educación meramente utilitarista o vista como medio para ganar dinero. El sujeto 8, que dirige una licenciatura de corte filosófico, responde en cambio que

....al ser una carrera que tiene, eh, un perfil muy específico, también es importante que la carrera tenga ciertas salidas laborales y que haya algunas materias que no son las principales, que no son las más [S8, P3].

\section{Pregunta 4: dilemas vividos respecto a la tensión entre educación para la renta y para la democracia}

En el área de Negocios los directores afirman enfrentar, más que dilemas, ciertas dificultades u obstáculos para formar a sus estudiantes en una visión ética profesional acorde con la educación para la democracia, porque

Los chicos que empiezan vienen más formados para una educación de la renta que para una educación de la democracia y nos cuesta mucho trabajo hacerlos que vean que el que emitan juicios críticos es algo importante para su desarrollo [S2, P4].

Este es el principal problema que declaran los directores prácticamente de todas las áreas que fueron entrevistados. Se expresa también en una dificultad para el trabajo en equipo, por una formación muy individualista y competitiva.

Sí, me parece que el principal está como en la... en esta idea más cientificista, ¿̇no?, que, sin excluir a las Humanidades, pues sigue habiendo paradigmas, posicionamientos o visiones en donde todavía el [interrupción por teléfono que suena] paradigma de un academicismo o de una alta, un alto valor a la formación científica, técnica; porque ni siquiera diría yo disciplinar, ¿no?, sino más aquello que es útil, que es funcionar, y que puede... o sea, hay una cierta tensión, creo que cada vez menor, y sobre todo en los programas orientados a la investigación educativa, pero sí en nuestros aspirantes, a buscar todavía una educación que... pues que sea funcional, que sea utilitaria, desde su propia motivación, incluso, ¿no?, "vengo para obtener un grado", "vengo para... eh, ganar más, para lograr un ascenso”, ¿no?, o sea, desde ahí hay una visión [S7, P4].

Esta dificultad parece común a la de los estudiantes de las carreras de Humanidades, según lo que vemos en esta respuesta. Se trata de una visión propia de educación para la renta, buscan aprendizajes funcionales con visiones utilitaristas que los lleven a obtener un ingreso, pero también, añade esta respuesta, la dificultad de una visión cientificista o academicista de las áreas de Humanidades que cierra muchas veces las posibilidades de una formación más integral. 


\section{Preguntas 5 a 7: narración de algún caso concreto que marque la tensión entre renta y democracia, visión sobre el cumplimiento de la misión de la universidad en un mercado centrado en la educación para la renta y áreas de oportunidad}

No, no. ¿Cómo te digo?... que las empresas inclusive me hablan o me escriben: "me espero y quiero uno de los tuyos porque me gusta más esa formación como muy... ese chip que traen” [S1, P6].

Esta es la respuesta de una directora del área de Negocios que habla de que las universidades que ofrecen un programa similar al suyo están muy orientadas hacia la educación para la renta y su programa es más integral y humanista, claramente orientado hacia la educación para la democracia.

Su respuesta señala que el mercado tampoco está tan satisfecho con una formación meramente técnica, sino que valora y busca a egresados de universidades que tengan características más completas en la formación y que desarrollen una visión más crítica, social y solidaria.

Coincide con esta visión el resto de los entrevistados, que plantean que en realidad la misión de la universidad, si bien es un reto porque la educación para la democracia es más completa y más compleja que la mera formación técnica de una educación para la renta, no presenta dificultades de gran calado porque los alumnos que llegan aprenden poco a poco a valorar la formación integral que se les ofrece y en general se comprometen con ella.

Los directores entrevistados coinciden también en que los retos para hacer mejor esta labor de educación para la democracia están básicamente en el diseño de currículos que integren mejor la formación en herramientas y elementos técnicos que les permitan insertarse en el mercado laboral cada vez mejor con el desarrollo de capacidades que los lleven a ser, como dice la misión universitaria, líderes que transformen a la sociedad.

\section{Conclusiones}

En términos generales se puede concluir que los directores académicos de esta universidad privada tienen un discurso que muestra una tendencia hacia el desarrollo de capacidades y la formación de sus estudiantes acorde a lo que Nussbaum llama educación para la democracia, y si bien integran elementos de capacitación técnica y científica necesarios para que al egresar puedan insertarse en un mercado laboral cada vez más competitivo, tienen claro que estas no son las únicas herramientas que se deben brindar a los futuros profesionistas.

Los directores hacen énfasis en la capacidad de pensar críticamente, en la sensibilidad para comprender al otro e incluirlo, y en el compromiso social para la transformación de las condiciones de vida de los grupos más vulnerables del país. 
No se percibe gran diferencia entre los directores académicos de licenciaturas del área de Negocios y los del área de Humanidades, salvo que los de Negocios hacen un poco más de énfasis en los elementos del mercado competido entre instituciones de educación superior y en herramientas de formación en matemáticas, inglés, software, etc., que los del área de Humanidades.

Esta convergencia de discursos muestra significados comunes entre los directores que seguramente se deben a la filosofía institucional y a los espacios de formación permanente y de actividades simbólicas que la comunidad universitaria realiza para reforzar esta identidad, que coincide con la visión que Nussbaum llama educación para la democracia y con el enfoque de capacidades que, como proponemos aquí, puede ser visto como una teoría ética profesional muy completa, útil y aplicable a nuestros contextos de formación universitaria.

Resulta interesante mencionar la constatación en las entrevistas de que solamente uno de los ocho directores que participaron conocía el pensamiento de Martha Nussbaum y sin embargo tienen bastante coincidencia sus respuestas desde la visión humanista de la universidad en que trabajan con las del planteamiento del desarrollo de capacidades y la educación para la democracia de la filósofa estadounidense.

Cabe la pregunta acerca de si los directores se han planteado con suficiente profundidad y sistematicidad la tensión entre ambas visiones de la educación, puesto que las respuestas en las preguntas relativas a dilemas y casos concretos vividos al respecto fueron las menos ricas.

La universidad en la que se realizó el estudio tiene, además de esta filosofía humanista, una cultura organizacional de corte empresarial muy arraigada también en toda la estructura universitaria, incluyendo la académica. Los directores académicos y los profesores parecen no notar tensiones, dilemas ni contradicciones serias entre estas dos culturas que corresponderían a los dos tipos de educación que plantea Nussbaum.

Sin embargo, en la universidad se vive continuamente la coexistencia en conflictos o tensiones implícitas entre exigencias y demandas de educación para la renta, como por ejemplo las exigencias de acreditación de programas y certificación de académicos y alumnos, los procesos administrativos que a veces ahogan la vida académica, etc.

De esto se desprende la conveniencia de seguir indagando este tema en la misma institución y de hacer algunas réplicas en instituciones que no tengan una filosofía humanista para ver si hay diferencias en los significados y vivencias de los directores que están a cargo de los programas de formación de los futuros profesionales de la educación del país. 


\section{ReFERENCIAS}

Álvarez-Gayou Jurgenson, J. L. (2012). Cómo hacer investigación cualitativa. Fundamentos y metodología. México: Paidós.

Beauchamp, T., y Childress, J. (2001). Principios de la ética biomédica. Barcelona: Masson.

Borrero, A. (2008). La universidad. Estudio sobre sus orígenes, dinámicas y tendencias. Bogotá: Pontificia Universidad Javeriana.

Brunner, J. J. (2006). Mercados universitarios: ideas, instrumentaciones y seis tesis en conclusión. Santiago: FONDECYT.

Cárcamo, H. (2005). Hermenéutica y análisis cualitativo. Cinta Moebio, (23), 204-216. Recuperado de: https:// revistaderechoeconomico.uchile.cl/index.php/ $\mathrm{CDM} /$ article/view/26081.

Cortina, A. (2000). Ética minima. Introducción a la filosofía práctica. Madrid: Ediciones Tecnos.

Cortina, A. (2000-2001). La manida palabra ética. Revista Contrastes. Recuperado de: https://www. uv.es/ fores/contrastes/quince/cortina.html.

El País (2010, jul. 7). Tras las buellas de Mabler. Cultura. Recuperado de: https://elpais.com/cultura/2010/07/06/ actualidad/1278367203_850215.html.

ETEA (s.f.). Tojeira: "las universidades están formando profesionales exitosos en sociedades fracasadas. ETEA/Universidad Loyola Andalucía. Recuperado de https://www. teol-granada.com/web/etea/noticia/-/journal_content/56_INSTANCE_m3CO/10192/933623.
Han, B.-Ch. (2017). La sociedad del cansancio. Barcelona: Herder.

Hernández Sampieri, R., Fernández Collado, C., y Baptista Lucio, P. (2010). Metodología de la investigación. México: McGraw-Hill.

Hortal, A. (1996). Siete tesis para repensar la ética profesional. Recuperado de: https://vdocuments.mx/siete-tesissobre-etica-profesional.html.

Hortal, A. (2002). Ética general de las profesiones. España: Desclée De Brouwer.

Lonergan, B. (1988). Método en teología. Salamanca: Sígueme. Morin, E. (2000). La mente bien ordenada. Barcelona: Seix Barral.

Morin, E. (2003). El método V. La bumanidad de la bumanidad. La identidad humana. Madrid: Cátedra.

Nussbaum, M. C. (1997). El cultivo de la humanidad. Una defensa clásica de la reforma en la educación liberal. Madrid: Paidós.

Nussbaum, M. C. (2010). Sin fines de lucro. Por qué la democracia necesita de las humanidades. Madrid: Katz.

Nussbaum, M. (2012). Creating capabilities. The buman development approach. Cambridge, MA: Harvard University Press. http://dx.doi.org/10.4159/harvard.9780674061200.

Sen, A. (1999) Development as freedom. Nueva York: Random House.

Sen, A. (2009). Teoría de la justicia. México: Taurus.

Cómo citar este artículo:

López-Calva, J. M., y Méndez Escarza, C. R. (2021). Enfoque de capacidades y ética profesional en la formación universitaria para la democracia: el caso de las carreras de negocios y humanidades de una universidad privada en México. IE Revista de Investigación Educativa de la REDIECH, 12, e1204. doi: 10.33010/ie_rie_rediech.v12i0.1204. 